OPEN ACCESS

Edited by:

Benjamin Frey,

University Hospital Erlangen, Germany

Reviewed by:

Zhang Xiaotian,

Peking University Cancer Hospital,

China

Abdolreza Sotoodeh Jahromi, Jahrom University of Medical

Sciences, Iran

*Correspondence:

Meng Qiu

qiumeng33@hotmail.com

${ }^{t}$ These authors share first authorship

Specialty section:

This article was submitted to

Cancer Immunity

and Immunotherapy,

a section of the journal

Frontiers in Immunology

Received: 29 July 2021 Accepted: 10 September 2021 Published: 29 September 2021

Citation:

Zhou W, Zhou Y, Yi C, Shu X,

Wei G, Chen X, Shen X

and Qiu M (2021) Case

Report: Immune and Genomic

Characteristics Associated With Hyperprogression in a Patient With

Metastatic Deficient Mismatch

Repair Gastrointestinal Cancer Treated With Anti-PD-1 Antibody.

Front. Immunol. 12:749204. doi: 10.3389/fimmu.2021.749204

\section{Case Report: Immune and Genomic Characteristics Associated With Hyperprogression in a Patient With Metastatic Deficient Mismatch Repair Gastrointestinal Cancer Treated With Anti-PD-1 Antibody}

\author{
Wenyue Zhou ${ }^{1 \dagger}$, Yuwen Zhou ${ }^{1 \dagger}$, Cheng $\mathrm{Yi}^{1+}$, Xinyao Shu ${ }^{1}$, Guixia Wei ${ }^{1}$, Xiaorong Chen ${ }^{1}$, \\ Xudong Shen ${ }^{2}$ and Meng Qiu ${ }^{1 *}$ \\ ${ }^{1}$ Department of Oncology, West China Hospital, Sichuan University, Chengdu, China, ${ }^{2}$ The Medical Department, \\ $3 D$ Medicines Inc., Shanghai, China
}

Microsatellite instability-high/deficient mismatch repair (MSI-H/dMMR) status of tumors is a distinct predictive biomarker of immune checkpoint inhibitors (ICls) for colorectal and non-colorectal cancer populations. The overall response rate (ORR) varies from approximately $40 \%$ to $60 \%$, indicating that nearly half of MSI-H tumors do not respond to ICls. The mechanism of response heterogeneity in MSI-H/dMMR cancers is unclear. Some patients who have been treated with ICls have developed a novel pattern of progression called hyperprogression, which is defined as unexpected accelerated tumor growth. No case of MSI-H/dMMR immunotherapy-associated hyperprogression has been reported in the literature. Here, we present the case of a patient with dMMR gastrointestinal cancer who suffered hyperprogressive disease (HPD) after treatment with nivolumab. We explored the potential mechanisms of HPD by clinical, immune, and genomic characteristics. Extremely high levels of serum LDH, low TMB and TILs, and the disruption of TGF $\beta$ signaling, may be related to hyperprogression.

Keywords: MSI-H/dMMR, immune checkpoint inhibitors, hyperprogression, gastrointestinal cancer, case report

\section{INTRODUCTION}

About $2-4 \%$ of diagnosed cancer patients are subtyped as "microsatellite instability-high (MSI-H)" or "deficient in mismatch repair (dMMR)" (1) Tumors with these genetic characteristics generally have exceptionally high tumor mutation burden and enriched infiltration of immune cells. These tumors are also known as "hot tumor" (2). Several vital clinical trials have confirmed that the microsatellite instability-high/deficient mismatch repair (MSI-H/dMMR) status is a distinct predictive biomarker of ICIs for colorectal (3) and non-colorectal $(1,4,5)$ cancer populations. The overall response rate (ORRs) varies from approximately $40 \%$ to $60 \%(1,3,6,7)$. Based on the 
different responses to immunotherapy, this phenomenon attracts significant interest in the heterogeneity and relevant mechanisms of MSI-H/dMMR cancer.

In contrast to chemotherapy and other types of anti-tumor therapy, immunotherapy-induced progression patterns are distinctive, including pseudoprogression and hyperprogression disease (HPD) (8). Pseudoprogression is defined as an initial increase in the tumor burden followed by a later or dissociated response (9). On the other hand, HPD manifests as rapid tumor growth combined with poor overall survival. The acknowledged mechanisms and valuable predictors of HPD remain largely unclear. There is evidence that some gene variations and clinical characteristics may be involved in the mechanism of HPD in different types of cancer. In non-small cell lung cancer and melanoma, patients who harbor MDM2/MDM4 amplification and EGFR alterations are reported to be more likely to develop hyperprogression (10-12). In contrast, those harboring the TP53 mutation without co-existence of STK11 or EGFR mutation have a high CD8 T cell density and, thus, a high response to ICIs (10). Approximately $1-6 \%$ of gastrointestinal cancer patients exhibit hyperprogression (HPD) (13). With respect to HPD in MSI-H/dMMR cancers, these phenomena and the mechanisms have not been reported.

Here, we present the first case of a patient with dMMR gastrointestinal cancer with primary resistance to immunotherapy, who experienced HPD after two doses of nivolumab. Nivolumab is a programmed death receptor-1 (PD-1)-blocking antibody and is approved for different types of cancer. Rapid hyperprogression was not observed. Pre-immunotherapy circulating tumor DNA (ctDNA) analysis using 150-gene panel next-generation sequencing, immunohistochemistry, multiplex fluorescence immunohistochemistry (mIHC), and blood inflammatory indexes were used to examine the immune microenvironment as well as the patient's genomic and clinical features. We also explored possible mechanisms of HPD. Furthermore, we identified the underlying mechanism and demonstrated the importance of personalized treatment for clinical practice.

\section{CASE DESCRIPTION}

\subsection{Case Presentation}

A 47-year-old male patient presented with constant abdominal pain for 4 months and was sent to the hospital for physical examination in October 2017. Computed tomography (CT) revealed multiple masses in the pancreatic body and both the lungs, with enlarged abdominal and supraclavicular lymph nodes, suggesting a heavy tumor load. The patient underwent supraclavicular lymph node dissection. Immunohistochemistry results suggested poorly differentiated metastatic adenocarcinoma. Based on CT imaging, the doctor-in-charge diagnosed that the tumor originated from the pancreas. Gemcitabine plus paclitaxel (Abraxane) chemotherapy was used as first-line treatment. Two months later, follow-up CT revealed tumor progression in both the lungs (Figure 1A). Positron-emission tomography-CT revealed increased intake of ${ }^{18} \mathrm{~F}$-fluorodeoxyglucose in the intestinal mesentery, cervicothoracic, abdominal lymph nodes, and both the lungs, indicating significant tumor load once again (Figure 1D). One enlarged abnormal cervical lymph node was punctured for examination because the patient refused dissection biopsy and immunohistochemistry was used to analyze the possible site of origin, tumor expression of MMR protein, and gene variation. Immunohistochemistry (IHC) of the second biopsy indicated that the tumor most likely originated from the gastrointestinal duct (IHC showed CDX2 positivity as shown in Figure 1B, microscopic magnification $\times 100$ ). A $5-10 \%$ loss of MLH1 and PMS2 protein expression and PD-L1 expression was also observed (Figures 1C, D, microscopic magnification $\times 100$ ), suggesting a $d M M R$ status in this patient. With a performance status (PS) score of 1 , nivolumab (200 mg every 2 weeks) was used as second-line treatment. Unfortunately, 1.5 month after the commencement of nivolumab (one dose of nivolumab), the patient experienced rapid deterioration in lung function, including obvious dyspnea and lethargy, and his performance status score deteriorated to 3. A follow-up chest CT scan confirmed rapid metastatic progression in the lungs and lymph nodes (Figure 1A). Moreover, laboratory tests revealed significant high serum lactate dehydrogenase (1360 IU/L, ranging from to 110-220 IU/L). Two days later, the patient died of respiratory failure.

\subsection{Diagnostic Assessments}

\subsubsection{Blood Test and Biopsy}

Serum lactate dehydrogenase and other blood tests were obtained the day before each treatment. The dissected lymph supraclavicular lymph node, and punctured cervical lymph node tissues were obtained under ultrasound and CT guidance, respectively.

\subsubsection{Diagnosis of HPD}

Currently, the most accepted definition of HPD is based on the study by Baptiste et al. (9) which detailed the following criteria: time to failure $<2$ months and TGK exp $\mathrm{TGK}_{\text {ref }}>2$. Tumor growth kinetics (TGK) are estimated to quantitatively assess the progression of the patient. According to Baptiste's definition, our patient's $\mathrm{TGK}_{\exp }$ and $\mathrm{TGK}_{\text {ref }}$ were 2.1 and 0.7.8, respectively, and the ratio $\left(\mathrm{TGK}_{\text {exp }}\right.$ : $\left.\mathrm{TGK}_{\text {ref }}\right)$ was 2.18. This value was calculated with reference to a single lesion, but the CT scan revealed a large number of newly developed lesions (Figure 1A). The tumor growth curve as shown in Figure 1E also indicated explosive tumor progression. The time to failure of the patient was 1.5 months. The ratio of $\mathrm{TGK}_{\exp }$ and $\mathrm{TGK}_{\text {ref }}$ was 2.18. As such, he met the definition of immunotherapy hyperprogression.

\subsubsection{Gene Analysis}

A 150-gene panel next generation sequencing (NGS) of circulating tumor DNA analysis (3D Medicines Co., Shanghai) before administration of nivolumab revealed six gene mutations with high frequency, including MLH1 p.N287Kfs (56.32\%), TCF7L2 p.K462Sfs (36.85\%), TP53 p.H179Y (41.4\%), ACVR2A p.K437Rfs (69.51\%), TGFBR2 p.K128Sfs (66.46\%), and TSC1 p.T635Rfs (5.41\%). Tumor NGS was detected via the punctured tissues. Three mutation genes were inspected (Figure 2D), including MLH1 p.N287Kfs (34.3\%), TP53 p.H179Y (38.4\%), and TSC1 p.T635Rfs (1.7\%). Tumor 


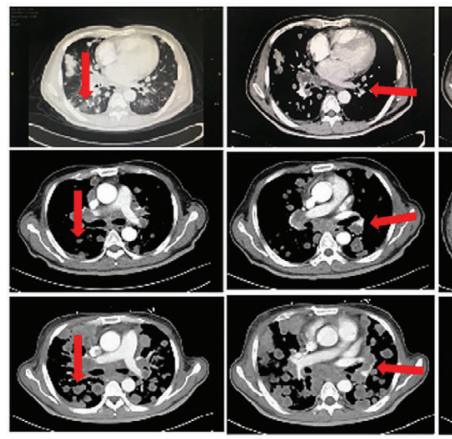

A

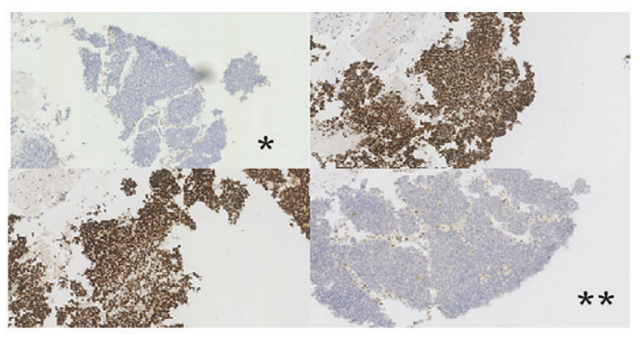

D

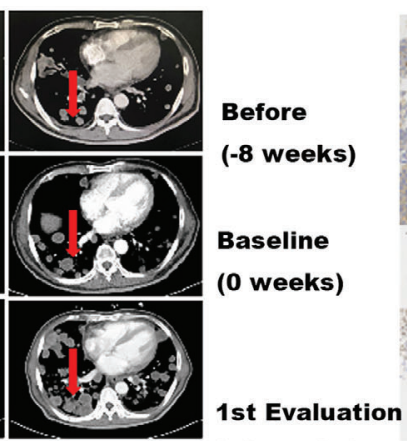

(+6 weeks)
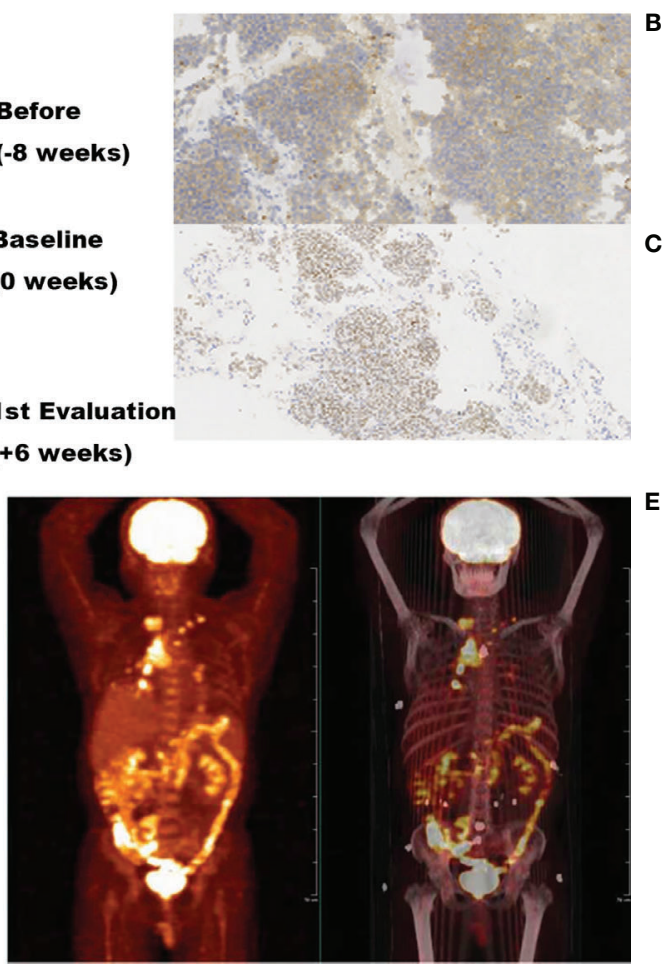

FIGURE 1 | Radiologic images before and after nivolumab showing distinct TGR differences. (A) Representative computed tomography scans of the patient's lung before, at baseline, and during treatment. (B) Second immunohistochemistry $(\mathrm{IHC})$ showing CDX2 positivity, indicating that the possible original tumor site may be the gastrointestinal duct. (C) Second IHC indicating a PD-L1 expression rate of 5-10\%. (D) Second IHC showing loss of MMR protein expression; *loss of MLH1 protein expression; **loss of expression of PMS2 protein. (E) Positron emission tomography/computed tomography (PET-CT) scan suggesting extensive tumor burden.

mutation burden (TMB) was also calculated. With a TMB of 7.26 Muts/Mb, the tumor was categorized as low TMB.

\subsubsection{Analysis of the Tumor Immune Microenvironment}

To further explore the possible refractory mechanism of HPD in this patient, the lymph node tissue tumor microenvironment (TME) of the patient was examined and PD-L1 protein expression was detected in the formalin-fixed, paraffinembedded (FFPE) punctured tumor tissues using the Dako PD-L1 IHC 22C3 pharmDx assay. The expression levels of CD8+, CD68+, CD56+ cells, pan cytokeratin, and HLA-DR were assessed and calculated using multiplex fluorescence immunohistochemistry (mIHC). Quantitative analysis of the tumor microenvironment showed that the degree of CD8+, CD68+, and CD56+ cells in the tumor stroma, and tumor region, were $0.59 \%$ and $1.28 \%$, respectively, along with $0.15 \%$, $0.30 \%$, and $0.63 \%$ and $0.49 \%$, respectively (Figure 2C).

\section{DISCUSSION}

This is the first reported case of HPD in MSI-H/dMMR gastrointestinal cancer, with no HPD-related gene mutation found in our patient. This is also the first report to demonstrate characteristics such as performance status, laboratory tests, TMB, TME, and tumor immune infiltrations (TILs) factors, and some gene alterations, to explain the case better.

Some clinical parameters are reliable predictive prognostic factors for patients with ICI-treated cancer. Studies have shown that high levels of serum lactate dehydrogenase (LDH), tumor burden, and poor performance status are independent baseline factors associated with hyperprogression $(14,15)$. According to the authors, LDH levels are strongly correlated with prognosis. The prognosis of patients within the normal range is within 16.1 months, but decreases to 2.3 months for those with $\mathrm{LDH}$ levels 2.5 times above the upper limit. The authors also demonstrated that low tumor burden and good performance status are also characteristics of favorable OS. As described, the patient had poor performance status, high $\mathrm{LDH}$ level, and a rather high tumor burden. The unfortunate outcome was consistent with previous conclusions.

In this case, the patient went through HPD although his TMB was 7.26 Muts/Mb (150-gene panel). TMB is stratified into two subtypes: low and high TMB. High TMB is usually defined as above the median burden based on whole exome sequencing (WES) or different gene panels (16). Optimal TMB cut-offs vary among different studies. However, levels lower than $10 \mathrm{Muts} / \mathrm{Mb}$ are generally categorized as low $(1,11,17)$. TMB is a reliable efficacy factor for immunotherapy $(11,18-21)$. Studies have 


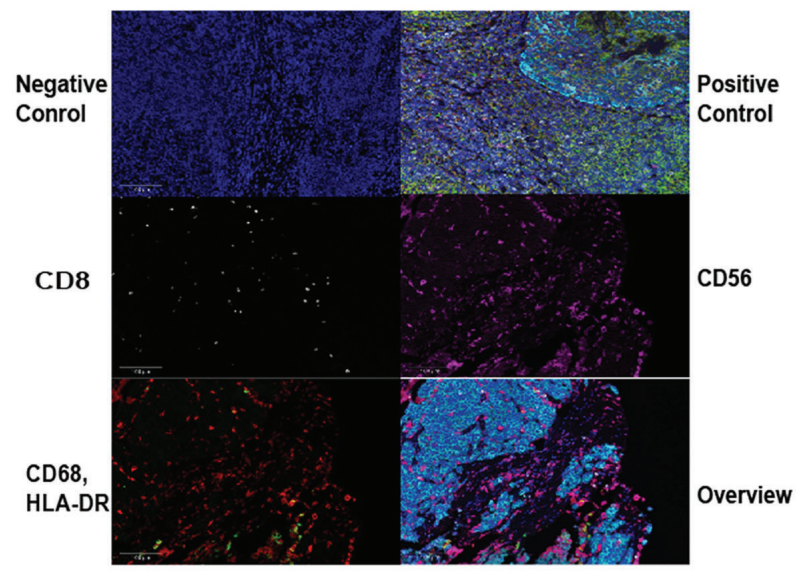

A

\begin{tabular}{|c|c|c|c|c|}
\hline \multirow{2}{*}{ Markers } & \multicolumn{2}{|c|}{ Intratumoral } & \multicolumn{2}{c|}{ stroma } \\
\cline { 2 - 5 } & Counts $/ \mathrm{mm}^{2}$ & $\%$ & Counts $/ \mathrm{mm}^{2}$ & $\%$ \\
\hline CD8 & 52 & 0.59 & 28 & 1.28 \\
\hline CD68+HLA-DR+ & 13 & 0.15 & 6 & 0.30 \\
\hline CD68+HLA-DR- & 4 & 0.05 & 7 & 0.34 \\
\hline CD56dim & 120 & 1.37 & 23 & 1.09 \\
\hline
\end{tabular}
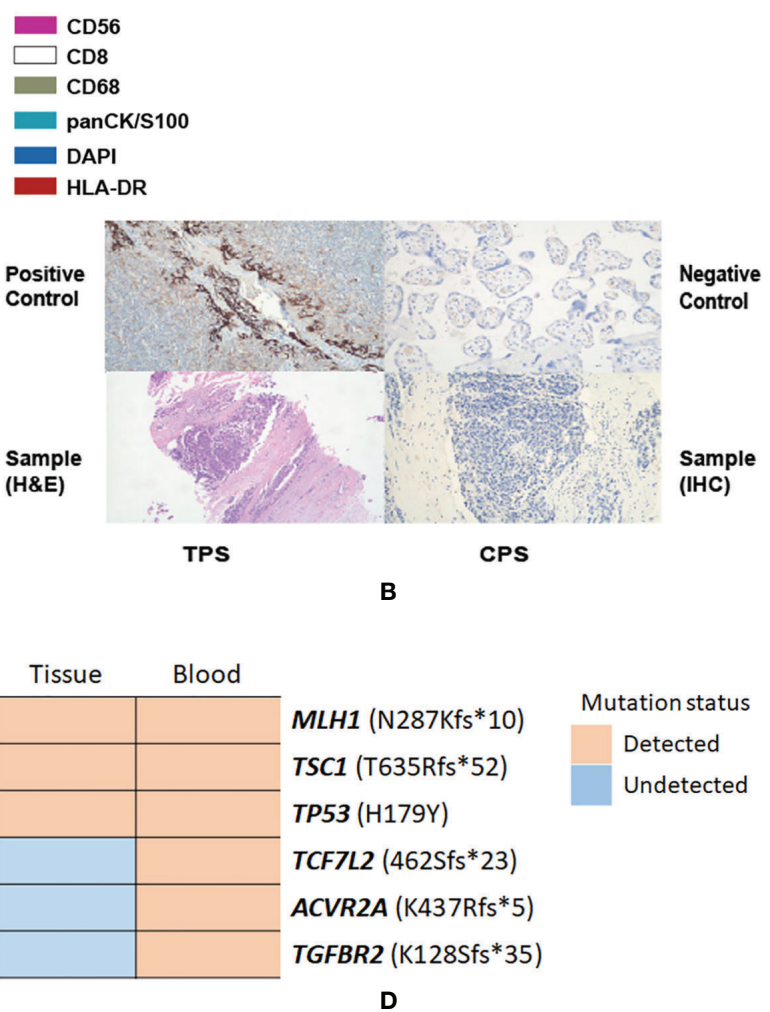

FIGURE 2 | Results showing tumor microenvironment and genomic mutation copies alteration in autopsy specimens. (A) Representative images of PanCK (pan cytokeratin), CD56, CD8, CD68, and HLA-DR as shown by $\mathrm{mlHC}$. Nuclei (blue) and counterstained by DAPI. Original magnification $\times 200$. (B) Tumor proportion score (TPS) and combined positive score (CPS). (C) Quantification results of TIL percentages by mIHC in the tumor stroma and margin. (D) Alteration of the genomic mutation copies before and after immunotherapy.

demonstrated that those with low TMB, as seen in this case, showed less favorable survival rate $(11,17,18,20-24)$. MSI-H/ dMMR tumors are identified with high clonality of nonsynonymous mutations (also high TMB), leading to the presence of robust altered proteins, known as neoantigens (25). Neoantigens are thought to be relevant to tumor control, as they can be targeted by the immune system, leading to a series of cytolytic activities of the immune cells $(1,3,25-27)$. With ORRs varies from $31.7 \%$ to $40 \%(3,28,29)$, nivolumab was approved for the treatment of melanoma, pancreatic, colorectal cancer, gastric cancer and so on. The intolerance and toxicities are mostly immune-mediated, along with infusion-related reactions and embryo-fatal adverse events. In this case, it is not hard to speculate that the low TMB reflected a minimal neoantigen, and was thus refractory to nivolumab. Hence, it is reasonable to assume that the heterogeneity of dMMR is partially responsible for the low levels of TMB and hyperprogression.

Heterogeneity of MSI-H/dMMR TILs was also observed by Harry H. Yoon et al. (30) TILs have been considered as positive prognostic predictive factors in tumor patients since the early 1900s. It is widely acknowledged that CD8+ TIL percentage is the most important predictive factor in immunotherapy (31). Compared with the MSS/pMMR status, CD3+ and CD8+ TILs are significantly more abundant in MSI-H/dMMR patients (30). In this case, as shown in Figures $\mathbf{2 A}, \mathbf{C}, \mathrm{CD} 8+$ lymphocytes, $\mathrm{CD} 68+$ macrophages, and CD56+ NK cell infiltration percentages were rather low. The PD-L1 assay (Figure 2A, original magnification $\times 200$ ) also revealed extremely low lymphocytes. According to Yoon et al., lower TILs in dMMR are associated with younger, non-smoking, and low histological grade patients. The tumor proportion score (TPS) and combined positive score (CPS) were also examined (Figure 2B, original magnification $\times 200$ ). The patient's TPS and CPS were $0 \%$ and $0 \%$, respectively (Dako 22C3 pharmDx, Santa Barbara, CA).

TME is classified into four types based on $\mathrm{T}$ cell infiltration and the presence of PD-L1 (32), and TME type III was categorized in this case. While $\mathrm{T}$ cell infiltration was observed in the patient, it appears that some unknown mechanisms prevented the effector $\mathrm{T}$ cells from functioning normally. A recent study also described that abnormal function of effector $\mathrm{T}$ cells may be a hindrance to their proliferation, execution, and maturation in immunotherapy-resistant TME type III patients (33). In addition, TILs in the tumor context have been proven to be a major positive prognostic predictive factor (34). Hence, low TIL percentages in patients cannot be neglected when attempting to elucidate the mechanism. Finally, although not yet listed as a 
negative factor of immunotherapy, some studies have indicated that TGF $\beta$ signaling could restrict T cells movement in TM. This then leads to a lack of response to anti-PD therapy and hyperprogression $(35,36)$.

Recent studies of TGF $\beta$ signaling may elucidate the possible mechanisms of hyperprogression. TGF $\beta$ is a bifunctional regulator that plays an important role in tumor initiation and progression, and deletions in the TGFBR2 gene loci can lead to TGF $\beta$ signaling disruption. Studies have confirmed that TGF $\beta$ derived epithelial-mesenchymal transition (EMT) can increase mesenchymal cells and lead to tissue fibrosis $(34,37)$. The pivotal role of TGF $\beta$ in tumor microenvironment (TME) fibrogenesis suggests that TGF $\beta$ signaling may restrict T cell movement and counteract anti-tumor immunity (35). TGF $\beta$ also plays a vital role in immunotherapy. By limiting the infiltration of inflammatory/immune cells, it could suppress CD8+ T cells and NK cell-mediated anti-tumor response (38). TGF $\beta$ inhibits $\mathrm{T}$ cell proliferation at the transcriptional level and represses their cytotoxic activity, resulting in tumor progression, and in some cases, hyperprogression. Xiong et al. found regulated TGF $\beta$ in the consistent sequencing of two patients with HPD (36). Unfortunately, our patient did not exhibit such a tendency (Figure 2D), but the small sample size in the study by Xiong et al. limited its credibility. Although TGF $\beta$ has not yet been confirmed as a negative regulator in immunotherapy, it is a new mechanism that merits further exploration. Apart from TGF $\beta$, deregulated WNT signaling could also increase resistance to TILs, as long as there is activation of epithelial-to-mesenchymal transition (EMT), and both methods could limit the infiltration of TILs, especially CD8+ lymph cells (39). In conclusion, TGF $\beta$ activation and WNT deregulation may explain the reason for nivolumab resistance and hyperprogression in this case.

Thus, HPD is a comprehensive-cause phenomenon. Studies have suggested that poor clinical characteristics, such as low TMB, heterogeneity of MSI-H/dMMR status, low levels of TILs, TGFBR2 alteration, and TME type III patients could develop

\section{REFERENCES}

1. Marabelle A, Le DT, Ascierto PA, Di Giacomo AM, Jesus-Acosta A D, Delord J-P, et al. Efficacy of Pembrolizumab in Patients With Noncolorectal High Microsatellite Instability or Mismatch Repair-Deficient Cancer, Results From the Phase II KEYNOTE-158 Study. J Clin Oncol (2019) 37:1-10. doi: 10.1200/ JCO.19.02105

2. Galon J, Bruni D. Approaches to Treat Immune Hot, Altered and Cold Tumours With Combination Immunotherapies. Nat Rev Drug Discov (2019) 18(3):197-218. doi: 10.1038/s41573-018-0007-y

3. Overman MJ, McDermott R, Leach JL, Lonardi S, Lenz H-J, Morse MA, et al. Nivolumab in Patients With Metastatic DNA Mismatch Repair-Deficient or Microsatellite Instability-High Colorectal Cancer (CheckMate 142): An Open-Label, Multicentre, Phase 2 Study. Lancet Oncol (2017) 18(9):118291. doi: 10.1016/S1470-2045(17)30422-9

4. Smith KN, Le DT, Durham JN. Mismatch-Repair Deficiency Predicts Response of Solid Tumors to PD-1 Blockade. Science (2017) 357:409-13. doi: $10.1126 /$ science.aan 6733

5. Le DT, Uram JN, Wang H, Bartlett BR, Kemberling H, Eyring AD, et al. PD-1 Blockade in Tumors With Mismatch-Repair Deficiency. N Engl J Med (2015) 372(26):2509-20. doi: 10.1056/NEJMoa1500596

6. Muro K, Chung HC, Shankaran V, Geva R, Catenacci D, Gupta S, et al. Pembrolizumab for Patients With PD-L1-Positive Advanced Gastric Cancer mechanism-unknown immune resistance. In this case, they may also lead to HPD.

\section{DATA AVAILABILITY STATEMENT}

The original contributions presented in the study are publicly available. This data can be found here: https://www.ncbi.nlm.nih. gov/sra/PRJNA756873.

\section{ETHICS STATEMENT}

This study was approved by the institutional review board of the West China Medical College of Sichuan University (ethical approval letter 20211077). The patient provided informed consent for his treatments, the collection and study of his blood and tissue samples. All procedures were conducted according to the Declaration of Helsinki. Written informed consent was obtained from the participant for the publication of this case report.

\section{AUTHOR CONTRIBUTIONS}

MQ conceived the idea of the article. WZ mainly takes charge of writing and figure-making. YZ and CY plays a guiding role in article revision, polish, genetic analysis, and ethical review. All authors contributed to the revision and discussion. All authors contributed to the article and approved the submitted version.

\section{ACKNOWLEDGMENTS}

We would like to thank the patient and his family for their understanding and 3D Medicine Company for their help with the study.

(KEYNOTE-012): A Multicentre, Open-Label, Phase 1b Trial. Lancet Oncol (2016) 17(6):717-26. doi: 10.1016/S1470-2045(16)00175-3

7. Le DT, Kim TW, Cutsem EV, Geva R, Jäger D, Hara H, et al. Phase II OpenLabel Study of Pembrolizumab in Treatment-Refractory, Microsatellite Instability-High/Mismatch Repair-Deficient Metastatic Colorectal Cancer: KEYNOTE-164. J Clin Oncol (2019) 38:11-9. doi: 10.1200/JCO.19.02107

8. Fuentes-Antras J, Provencio M, Diaz-Rubio E. Hyperprogression as a Distinct Outcome After Immunotherapy. Cancer Treat Rev (2018) 70:16-21. doi: 10.1016/j.ctrv.2018.07.006

9. Kas B, Talbot H, Ferrara R, Richard C, Lamarque JP, Pitre-Champagnat S, et al. Clarification of Definitions of Hyperprogressive Disease During Immunotherapy for Non-Small Cell Lung Cancer. JAMA Oncol (2020) 6 (7):1039-46. doi: 10.1001/jamaoncol.2020.1634

10. Biton J, Mansuet-Lupo A, Pecuchet N, Alifano M, Ouakrim H, Arrondeau J, et al. TP53, STK11, and EGFR Mutations Predict Tumor Immune Profile and the Response to Anti-PD-1 in Lung Adenocarcinoma. Clin Cancer Res (2018) 24(22):5710-23. doi: 10.1158/1078-0432.CCR-18-0163

11. Samstein RM, Lee CH, Shoushtari AN, Hellmann MD, Shen R, Janjigian YY, et al. Tumor Mutational Load Predicts Survival After Immunotherapy Across Multiple Cancer Types. Nat Genet (2019) 51(2):202-6. doi: 10.1038/s41588018-0312-8

12. Gatalica Z, Xiu J, Swensen J, Vranic S. Comprehensive Analysis of Cancers of Unknown Primary for the Biomarkers of Response to Immune Checkpoint 
Blockade Therapy. Eur J Cancer (2018) 94:179-86. doi: 10.1016/ j.ejca.2018.02.021

13. Champiat S, Dercle L, Ammari S, Massard C, Hollebecque A, Postel-Vinay S, et al. Hyperprogressive Disease Is a New Pattern of Progression in Cancer Patients Treated by Anti-PD-1/PD-L1. Clin Cancer Res (2017) 23(8):1920-8. doi: 10.1158/1078-0432.CCR-16-1741

14. Weide B, Martens A, Hassel JC, Berking C, Postow MA, Bisschop K, et al. Baseline Biomarkers for Outcome of Melanoma Patients Treated With Pembrolizumab. Clin Cancer Res (2016) 22(22):5487-96. doi: 10.1158/10780432.CCR-16-0127

15. Kim JY, Lee KH, Kang J, Borcoman E, Saada-Bouzid E, Kronbichler A, et al. Hyperprogressive Disease During Anti-PD-1 (PDCD1)/PD-L1 (CD274) Therapy: A Systematic Review and Meta-Analysis. Cancers (Basel) (2019) 11(11):1699. doi: 10.3390/cancers11111699

16. Rizvi NA, Hellmann MD, Snyder A, Kvistborg P, Makarov V, Havel JJ, et al. Mutational Landscape Determines Sensitivity to PD-1 Blockade in Non-Small Cell Lung Cancer. Science (2016) 348(6230):124-8. doi: 10.1126/science. aaa1348

17. Chida K, Kawazoe A, Kawazu M, Suzuki T, Nakamura Y, Nakatsura T, et al. A Low Tumor Mutational Burden and PTEN Mutations are Predictors of a Negative Response to PD-1 Blockade in MSI-H/dMMR Gastrointestinal Tumors. Clin Cancer Res (2021) 27:3714-24. doi: 10.1158/1078-0432.CCR-21-0401

18. Leslie M. High TMB Predicts Immunotherapy Benefit. Cancer Discov (2018) 8(6):668. doi: 10.1158/2159-8290.CD-NB2018-048

19. Saâda-Bouzid E, Defaucheux C, Karabajakian A, Coloma VP, Servois V, Paoletti X, et al. Hyperprogression During Anti-PD-1/PD-L1 Therapy in Patients With Recurrent and/or Metastatic Head and Neck Squamous Cell Carcinoma. Ann Oncol (2017) 28(7):1605-1611. doi: 10.1093/annonc/ $\operatorname{mdx} 178$

20. Cristescu R, Mogg R, Ayers M, Albright A, Murphy E, Yearley J, et al. Pan-Tumor Genomic Biomarkers for PD-1 Checkpoint Blockade-Based Immunotherapy. Science (2018) 362(6411):eaar3593. doi: 10.1126/science.aar3593

21. Jones S, Anagnostou V, Lytle K, Parpart-Li S, Nesselbush M, Riley DR, et al. Personalized Genomic Analyses for Cancer Mutation Discovery and Interpretation. Cancer (2015) 7(283):283ra53. doi: 10.1126/scitranslmed.aaa7161

22. Liu L, Bai X, Wang J, Tang XR, Wu DH, Du SS, et al. Combination of TMB and CNA Stratifies Prognostic and Predictive Responses to Immunotherapy Across Metastatic Cancer. Clin Cancer Res (2019) 25(24):7413-23. doi: 10.1158/1078-0432.CCR-19-0558

23. Petrelli F, Ghidini M, Ghidini A, Tomasello G. Outcomes Following Immune Checkpoint Inhibitor Treatment of Patients With Microsatellite InstabilityHigh Cancers: A Systematic Review and Meta-Analysis. JAMA Oncol (2020) 6 (7):1068-71. doi: 10.1001/jamaoncol.2020.1046

24. Lu S, Stein JE, Rimm DL, Wang DW, Bell JM, Johnson DB, et al. Comparison of Biomarker Modalities for Predicting Response to PD-1/PD-L1 Checkpoint Blockade: A Systematic Review and Meta-Analysis. JAMA Oncol (2019) 5 (8):1195-204. doi: 10.1001/jamaoncol.2019.1549

25. Baretti M, Le DT. DNA Mismatch Repair in Cancer. Pharmacol Ther (2018) 189:45-62. doi: 10.1016/j.pharmthera.2018.04.004

26. Yang W, Lee KW, Srivastava RM, Kuo F, Krishna C, Chowell D, et al. Immunogenic Neoantigens Derived From Gene Fusions Stimulate T Cell Responses. Nat Med (2019) 25(5):767-75. doi: 10.1038/s41591-019-0434-2

27. Schumacher TN, Schreiber RD. Neoantigens in Cancer Immunotherapy. Science (2015) 348(6230):69-74. doi: 10.1126/science.aaa4971

28. Weber JS, D’Angelo SP, Minor D, Hodi FS, Gutzmer R, Neyns B, et al. Nivolumab Versus Chemotherapy in Patients With Advanced Melanoma Who Progressed After Anti-CTLA-4 Treatment (CheckMate 037): A Randomised, Controlled, Open-Label, Phase 3 Trial. Lancet Oncol (2015) 16 (4):375-84. doi: 10.1016/S1470-2045(15)70076-8
29. Robert C, Long GV, Brady B, Dutriaux C, Maio M, Mortier L, et al. Nivolumab in Previously Untreated Melanoma Without BRAF Mutation. $N$ Engl J Med (2015) 372(4):320-30. doi: 10.1056/NEJMoa1412082

30. Yoon HH, Shi Q, Heying EN, Muranyi A, Bredno J, Ough F, et al. Intertumoral Heterogeneity of $\mathrm{CD} 3(+)$ and $\mathrm{CD} 8(+)$ T-Cell Densities in the Microenvironment of DNA Mismatch-Repair-Deficient Colon Cancers: Implications for Prognosis. Clin Cancer Res (2019) 25(1):125-33. doi: 10.1158/1078-0432.CCR-18-1984

31. Chiou SH, Sheu BC, Chang WC, Huang SC, Hong-Nerng H. Current Concepts of Tumor-Infiltrating Lymphocytes in Human Malignancies. J Reprod Immunol (2005) 67(1-2):35-50. doi: 10.1016/j.jri.2005.06.002

32. Taube JM, Anders RA, Young GD, Xu H, Sharma R, McMiller TL, et al. Colocalization of Inflammatory Response With B7-H1 Expression in Human Melanocytic Lesions Supports an Adaptive Resistance Mechanism of Immune Escape. Sci Trans Med (2012) 4(127):127ra37. doi: 10.1126/ scitranslmed.3003689

33. Kim TK, Herbst RS, Chen L. Defining and Understanding Adaptive Resistance in Cancer Immunotherapy. Trends Immunol (2018) 39(8):62431. doi: 10.1016/j.it.2018.05.001

34. Bremnes RM, Busund L-T, Kilvær TL, Andersen S, Richardsen E, Paulsen EE, et al. The Role of Tumor-Infiltrating Lymphocytes in Development, Progression, and Prognosis of Non-Small Cell Lung Cancer. J Thorac Oncol (2016) 11(6):789-800. doi: 10.1016/j.jtho.2016.01.015

35. Mariathasan S, Turley SJ, Nickles D, Castiglioni A, Yuen K, Wang Y, et al. TGFbeta Attenuates Tumour Response to PD-L1 Blockade by Contributing to Exclusion of T Cells. Nature (2018) 554(7693):544-8. doi: 10.1038/nature25501

36. Xiong D, Wang Y, Singavi AK, Mackinnon AC, George B, You M. Immunogenomic Landscape Contributes to Hyperprogressive Disease After Anti-PD-1 Immunotherapy for Cancer. iScience (2018) 9:258-77. doi: 10.1016/j.isci.2018.10.021

37. Morikawa M, Derynck R, Miyazono K. TGF-Beta and the TGF-Beta Family: Context-Dependent Roles in Cell and Tissue Physiology. Cold Spring Harb Perspect Biol (2016) 8(5):a021873. doi: 10.1101/cshperspect.a021873

38. Yang L, Pang Y, Moses HL. TGF-Beta and Immune Cells: An Important Regulatory Axis in the Tumor Microenvironment and Progression. Trends Immunol (2010) 31(6):220-7. doi: 10.1016/j.it.2010.04.002

39. Galluzzi L, Spranger S, Fuchs E, Lopez-Soto A. WNT Signaling in Cancer Immunosurveillance. Trends Cell Biol (2019) 29(1):44-65. doi: 10.1016/ j.tcb.2018.08.005

Conflict of Interest: XDS is employed by 3D Medicines Inc.

The remaining authors declare that the research was conducted in the absence of any commercial or financial relationships that could be construed as a potential conflict of interest.

Publisher's Note: All claims expressed in this article are solely those of the authors and do not necessarily represent those of their affiliated organizations, or those of the publisher, the editors and the reviewers. Any product that may be evaluated in this article, or claim that may be made by its manufacturer, is not guaranteed or endorsed by the publisher.

Copyright $\odot 2021$ Zhou, Zhou, Yi, Shu, Wei, Chen, Shen and Qiu. This is an openaccess article distributed under the terms of the Creative Commons Attribution License (CC BY). The use, distribution or reproduction in other forums is permitted, provided the original author(s) and the copyright owner(s) are credited and that the original publication in this journal is cited, in accordance with accepted academic practice. No use, distribution or reproduction is permitted which does not comply with these terms. 